\title{
Thermal Cycling of Thermal Control Paints on Carbon- Carbon and Carbon-Polyimide Composites
}

\author{
Donald A. Jaworske* \\ NASA Glenn Research Center, Cleveland, Ohio, 44135
}

\begin{abstract}
Carbon-carbon composites and carbon-polyimide composites are being considered for space radiator applications owing to their light weight and high thermal conductivity. For those radiator applications where sunlight will impinge on the surface, it will be necessary to apply a white thermal control paint to minimize solar absorptance and enhance infrared emittance. Several currently available white thermal control paints were applied to candidate carbon-carbon and carbon-polyimide composites and were subjected to vacuum thermal cycling in the range of $-100{ }^{\circ} \mathrm{C}$ to $+277{ }^{\circ} \mathrm{C}$. The optical properties of solar absorptance and infrared emittance were evaluated before and after thermal cycling. In addition, adhesion of the paints was evaluated utilizing a tape test. The test matrix included three composites: resin-derived carbon-carbon and vapor infiltrated carbon-carbon, both reinforced with pitch-based P-120 graphite fibers, and a polyimide composite reinforced with T-650 carbon fibers, and three commercially available white thermal control paints: AZ-93, Z-93-C55, and YB-71P.
\end{abstract}

\section{Nomenclature}

$\alpha=$ solar absorptance

$\varepsilon \quad=$ infrared emittance

AMO = air mass zero

$\mathrm{n} / \mathrm{a}=$ not applicable

\section{Introduction}

$\mathrm{P}$ itch-based carbon-carbon composites and carbon-polyimide composites are being considered for use in space radiator applications, owing to their high thermal conductivity and low mass. Carbon-polyimide composites are particularly attractive owing to their ease of construction compared to their carbon-carbon composite alternative. Such composites, however, are black at the time of construction and absorb sunlight adding to the heat load of the radiator. To alleviate this problem, the surface of the composite must be coated with a white thermal control paint having the combined properties of low solar absorptance, $\alpha$, and high infrared emittance, $\varepsilon$. Several thermal control paints are available for this purpose, however, they were originally designed for use on radiators having aluminum facesheets. Hence, it is unclear whether the white thermal control paints developed in the past are suitable for use on recently developed carbon-carbon and carbon-polyimide composites.

The two issues of concern for utilizing white thermal control paints on these composites are: maintaining optical properties and adhesion. The thermal control paint optical properties as-deposited on composites are expected to be similar to the optical properties as-deposited on aluminum radiator facesheets owing to the thickness of the paint. Adhesion, however, is of concern because of the substantially different surface chemistry of the composite compared to aluminum. The coefficient of thermal expansion of a composite facesheet is also substantially different than that of an aluminum facesheet, suggesting that thermal cycling of the composite and coating combination may exacerbate adhesion problems.

Coupons of candidate composite and thermal control paint combinations were prepared. The optical properties of solar absorptance and infrared emittance were evaluated before and after thermal cycling to evaluate optical properties durability. Adhesion testing based on a variation of the ASTM D-3359 tape test was also conducted on both the as-received painted composites and composites that were subjected to thermal cycling. This paper

\footnotetext{
* Senior Research Engineer, Electro-Physics Branch, Mail Stop 309-2, 21000 Brookpark Road, Cleveland, OH, 44135, Non-Member.
} 
summarizes the optical properties and adhesion research conducted on the various combinations of white thermal control paints and composites.

\section{Materials and Methods}

\section{A. Composite Coupons}

Three composites were considered in this study, two carbon-carbon composites and one carbon-polyimide composite. Both types of carbon-carbon composites were reinforced with pitch-based P-120 graphite fibers. One set of specimens were prepared by C-CAT (Carbon-Carbon Advanced Technologies), Inc., Fortworth, TX by a resin infiltration and pyrolysis process, designated C-CAT. Another set of specimens was obtained from Goodrich Corp., Santa Fe Springs, CA. These composites were prepared by high temperature pitch impregnation and pyrolysis followed by carbon densification using a chemical vapor infiltration (CVI) process, designated Goodrich. Both types of composite plates were machined into $5 \mathrm{~cm}$ x $5 \mathrm{~cm}$ pieces, cleaned in acetone using an ultrasonic bath, and dried in an oven before application of coatings. The carbon-polyimide was manufactured at NASA Glenn Research Center as part of an ongoing research and development effort and utilized PAN-based T-650 fibers, designated carbon-polyimide composite. Three thermal control paints were considered in this study. One thermal control paint, manufactured by AZ Technology, was a non conductive formulation using zinc oxide pigment in a potassium silicate binder, designated AZ-93. As part of the deposition process for this paint, an epoxy primer was utilized to form the interface between composite and thermal control paint. Use of the epoxy primer, in this case only, limited the upper use temperature to $177^{\circ} \mathrm{C}$. The second thermal control paint, manufactured by Alion, was another formulation using a zinc oxide pigment in a potassium silicate binder, designated Z-93-C55. This version of white thermal control paint included a conductive oxide dopant to provide some electrical conductivity to the coating. Alion purposely abraded the surface of the composite coupons, utilizing a proprietary method, to promote adhesion between composite and thermal control paint. The polyimide matrix limited the upper use temperature for these coupons to $277^{\circ} \mathrm{C}$, and would also limit the upper use temperature of any radiator that might be made from this material. This temperature is acceptable for some radiator concepts while other concepts require even greater temperatures. The third thermal control paint, also manufactured by Alion, was a formulation using a zinc-orthotitanate pigment in a potassium silicate binder, designated YB-71P. Again, the surface of each composite was purposely abraded to promote adhesion. All three thermal control paints were applied utilizing a spray on technique.

\section{B. Optical Properties Measurements}

Solar absorptance was evaluated utilizing a Perkin-Elmer Lambda-19 spectrophotometer equipped with a $15 \mathrm{~cm}$ diameter integrating sphere. Total reflectance obtained in the wavelength range of 250 to $2500 \mathrm{~nm}$ was subtracted from unity, and weighted with respect to the air mass zero (AM0) solar spectrum to yield solar absorptance. Solar absorptance, as calculated with this method, is temperature independent. Infrared emittance was evaluated utilizing a Surface Optics Corporation SOC-400t portable infrared reflectometer equipped with barrel optics. Total reflectance obtained in the wavelength range of 2 to 25 microns was subtracted from unity, and weighted with respect to the blackbody spectrum at room temperature to yield infrared emittance. Although room temperature emittance values are utilized here for comparison, this method can also be used for identifying emittance at temperatures other than room temperature, providing the appropriate blackbody spectrum is used during the weighting step in the calculation, i.e. infrared emittance, as calculated with this method, is temperature dependent.

\section{Vacuum Thermal Cycling}

Vacuum thermal cycling was accomplished utilizing a bell jar, as shown in Figure 1. The bell jar was pumped by an oil diffusion pump backed by a roughing pump and typically achieved a vacuum of $9 \times 10^{-5} \mathrm{~Pa}$. Above the diffusion pump was a cold trap of water-cooled and liquid nitrogen-cooled baffles. Heating and cooling of a single coupon occurred through radiative processes. The bell jar was equipped with a ceramic heater and liquid nitrogencooled cold pocket. A swing arm under the control of a programmable logic controller swung each coupon from the heater to the cold pocket and back, repeatedly, based on pre-selected temperature set points. The programmable logic controller also monitored the number of cycles accrued. A complete cycle took approximately four hours, depending on temperature set points, the mass of the coupon, and its optical properties. The set points chosen for this study were nominally $-100{ }^{\circ} \mathrm{C}$ to $+177^{\circ} \mathrm{C}$ for the coupons containing the epoxy primer, and $-100{ }^{\circ} \mathrm{C}$ to $+277^{\circ} \mathrm{C}$ for all other coupons.

In some radiator applications, the radiator is expected to operate at its designated use temperature without interruption, with the exception of rare or unexpected events which may allow the radiator to cool. Such cooling events are expected to be small in number. Hence the coupons studied here were cycled for a small number of 
cycles. As mentioned above, the high end temperatures selected for thermal cycling were based on the coupon's materials properties. The low end temperature selected for thermal cycling was based on an unexpected cooling event along with some margin.

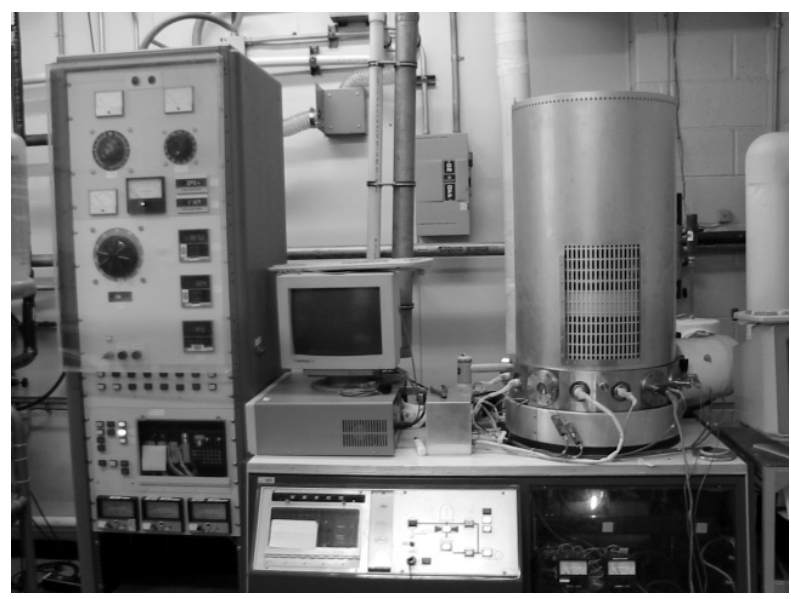

Figure 1. Vacuum Thermal Cycling Facility

Coupon temperature was monitored by a Keithley programmable thermometer, thus keeping a record of sample temperature as a function of time. Two thermocouples were placed on the back side of the sample. Coupon temperature was monitored and compared to the set point temperatures by the programmable logic controller thermocouple approximately once per second, keeping the sample from going outside of the set point temperatures. Coupon temperature was also monitored by the Keithley programmable thermometer thermocouple, at a sampling rate of once every five minutes. As will be described later, the set points were softened as needed to accommodate changes in optical properties that occurred during thermal cycling. Although the programmable logic controller was set for ten cycles, unexpected shut downs truncated some runs while restarts added more cycles to others. A summary of the number of thermal cycles delivered to each coupon is provided in Table 1.

Table 1. Summary of the number of thermal cycles delivered to each composite coupon.

\begin{tabular}{|l|c|c|c|}
\hline & $\begin{array}{c}\text { C-CAT } \\
\text { Carbon-Carbon }\end{array}$ & $\begin{array}{c}\text { Goodrich } \\
\text { Carbon-Carbon }\end{array}$ & $\begin{array}{c}\text { GRC } \\
\text { Carbon-Polyimide }\end{array}$ \\
\hline AZ-93 & 12 cycles & 14 cycles & 7 cycles \\
\hline Z-93-C55 & 6 cycles & 12 cycles & 8 cycles \\
\hline YB-71P & 3 cycles & 10 cycles & 14 cycles \\
\hline
\end{tabular}

\section{Adhesion Testing}

Adhesion testing was conducted on pristine and thermal-cycled samples. The adhesion testing was based on a variation of ASTM D-3359 test method A, the X-cut tape test. ${ }^{1}$ This variation was used based on past experience in evaluating Z-93-P thermal control paint. In test method A, a $5 \mathrm{~cm}$ " $X$ " is scribed onto the coupon with a scalpel, a $2.54 \mathrm{~cm}$ wide pressure sensitive standard tape is applied on top of the " $\mathrm{X}$ " using a rubber eraser. The tape is then removed rapidly within 90 seconds of its application, pulling back at an angle as close to $180^{\circ}$ as possible. A six point scale is provided in the standard to yield a rating for the degree of adhesion. Table 2 summarizes the rating scale. The variation used here utilized two $5 \mathrm{~cm}$ parallel lines scribed onto the coupon approximately $1.5 \mathrm{~cm}$ apart. A scalpel was used, cutting through the paint to the substrate in a single cut. The tape was applied normal to the two scribed lines with four passes of a rubber roller. Furthermore, the tape was removed rapidly after 30 seconds of its application by pulling back at an angle as close to $90^{\circ}$ as possible. The tape selected for use in the tape test was a 3M 250 paper backed masking tape, the same type of tape as was used in previous evaluation of Z-93-P thermal control paint. 
Table 2. ASTM D-3359 Rating Scale

\begin{tabular}{|c|l|}
\hline Rating & \multicolumn{1}{|c|}{ Description } \\
\hline 5A & No Peeling or removal \\
\hline 4A & Trace peeling or removal along incisions \\
\hline 3A & Jagged removal along incisions up to 1.8 mm on either side \\
\hline 2A & Jagged removal along most of incisions up to $3.2 \mathrm{~mm}$ on either side \\
\hline 1A & Removal from most of the area under the tape \\
\hline 0A & Removal beyond the area under the tape \\
\hline
\end{tabular}

\section{Results and Discussion}

The as-painted substrates appeared to have good coverage, with the paint appearing pale white for the Z-93-C55 to bright white for the AZ-93 and YB-71P. Paint thickness was estimated to be $0.08 \mathrm{~mm}$. Most as-painted coupons were featureless, however, some of the YB-71P coupons did have slight print through. Each vendor reported that applying their paint to the composites was a challenge, particularly the carbon-polyimide substrate. The post thermal cycling appearance varied from a similar good coverage to total adhesion failure. Photographs of each sample were obtained, next to sister samples, to show the degradation in appearance and are shown in Figure 2.
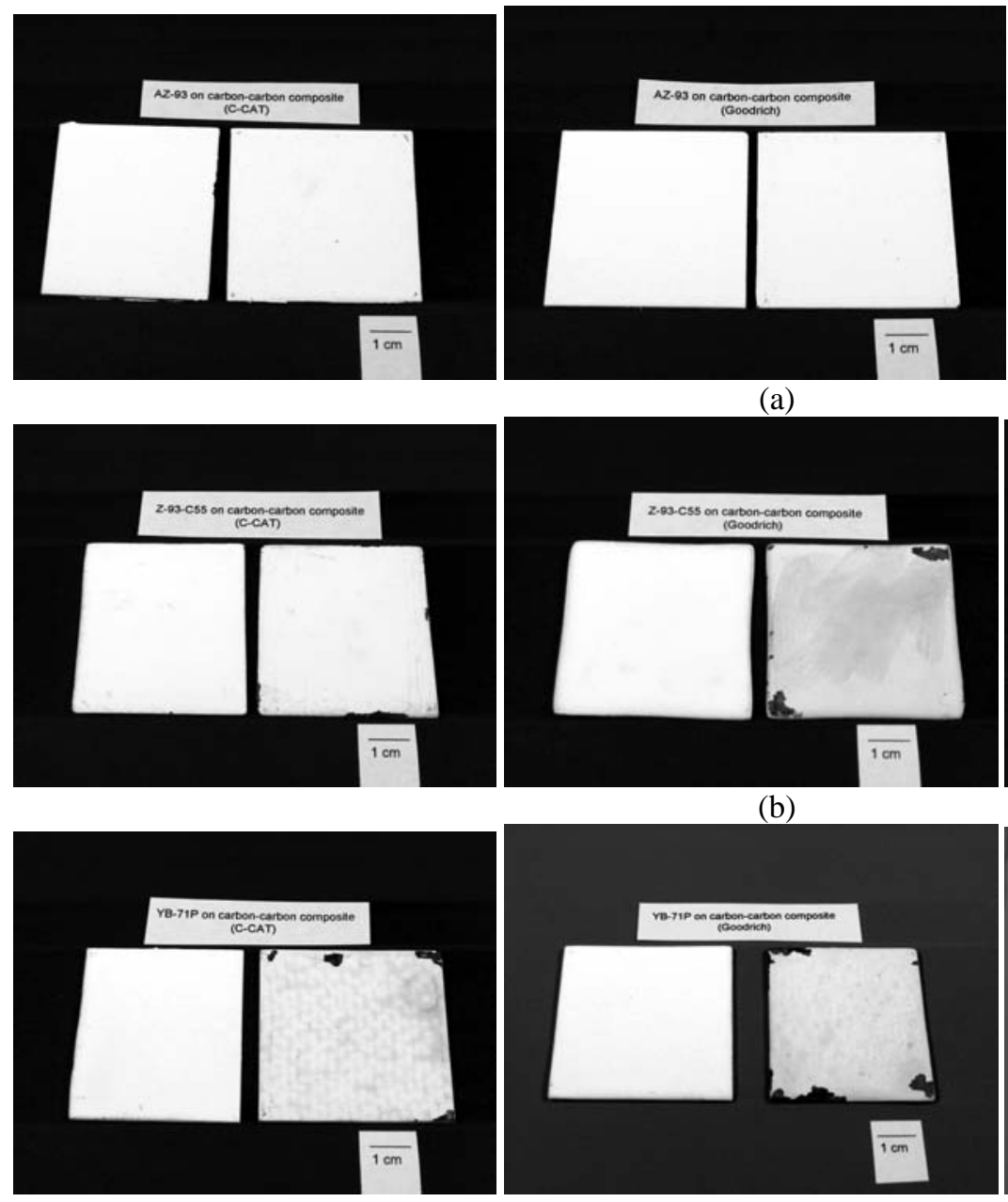

(a)

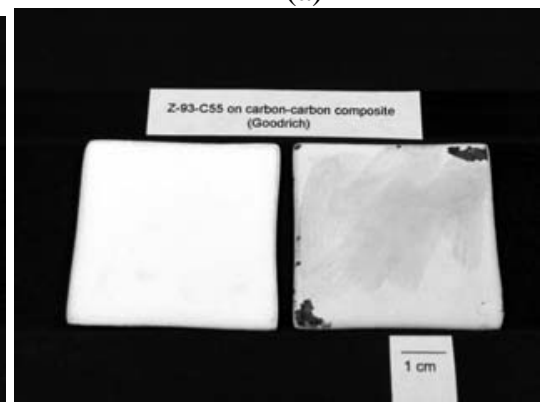

(b)

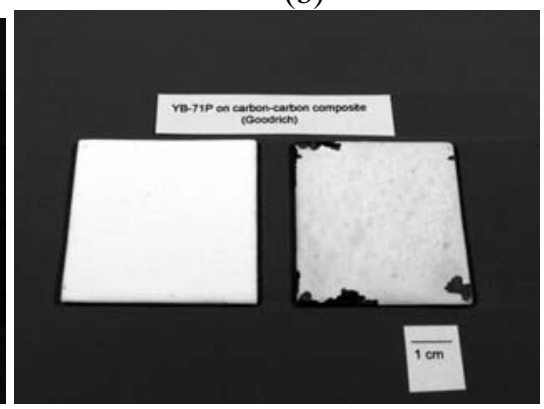

(c)
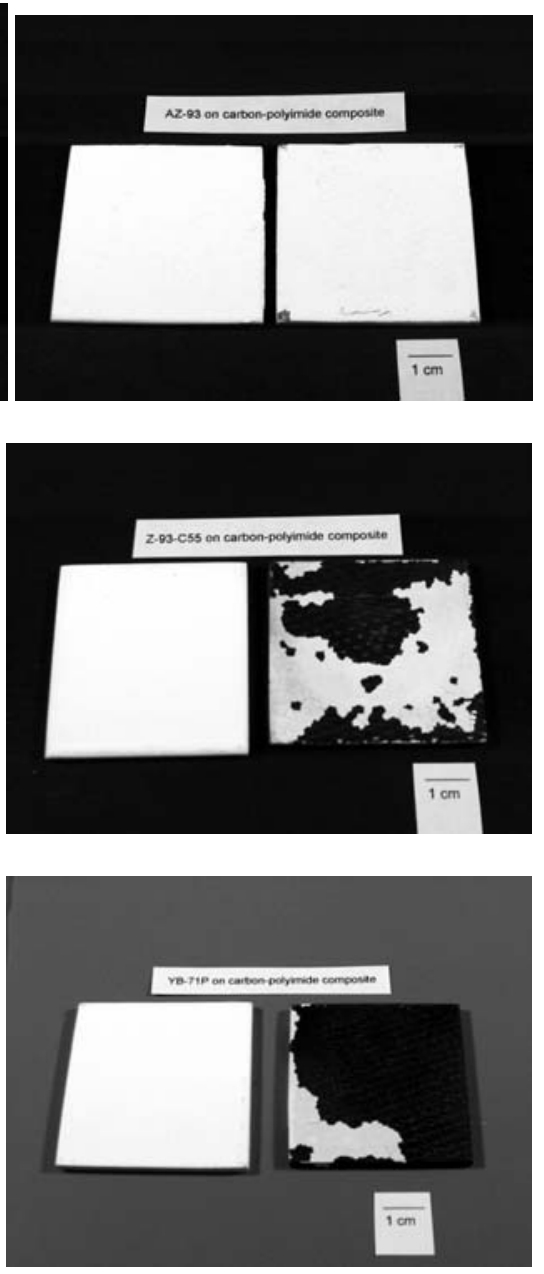

Figure 2. Photographs of pristine (left) and exposed (right) sample coupons obtained after thermal cycling: a) AZ-93 on C-CAT, Goodrich, and carbon-polyimide composites, b) Z-93-C55 on C-CAT, Goodrich, and carbonpolyimide composites, and c) YB-71P on C-CAT, Goodrich, and carbon-polyimide composites. 


\section{A. Thermal Cycling}

During the operation of the vacuum thermal cycling facility, it was found that the ceramic heater had ample heating capacity and easily provided sufficient heating to achieve the required hot end temperature of $277^{\circ} \mathrm{C}$. However, during cooling, it was found that care was needed in isolating the sample thermally to achieve the required low end temperature of $-100^{\circ} \mathrm{C}$. Improvements made to the system include a low thermal conductance connection to the swing arm holding the sample and installation of an aluminum "curtain" to close the cold pocket from its surroundings. Even with such improvements, changes in the optical properties over the course of thermal cycling occasionally required that the set points controlling high end or low end temperature needed to be changed slightly during the course of the thermal cycling program. As a consequence of the time needed to heat and cool the samples, much of the vacuum thermal cycling was accomplished during unattended operation. The programmable logic controller monitored sample temperature in real time to determine the successful meeting of high or low temperature set points, however, the programmable thermometer used to capture the thermal cycling profile collected data every 5 minutes. An example of a thermal cycling profile is shown in Figure 3. Note the softening of the cold end set point, as a consequence of deteriorating thermal optical properties.

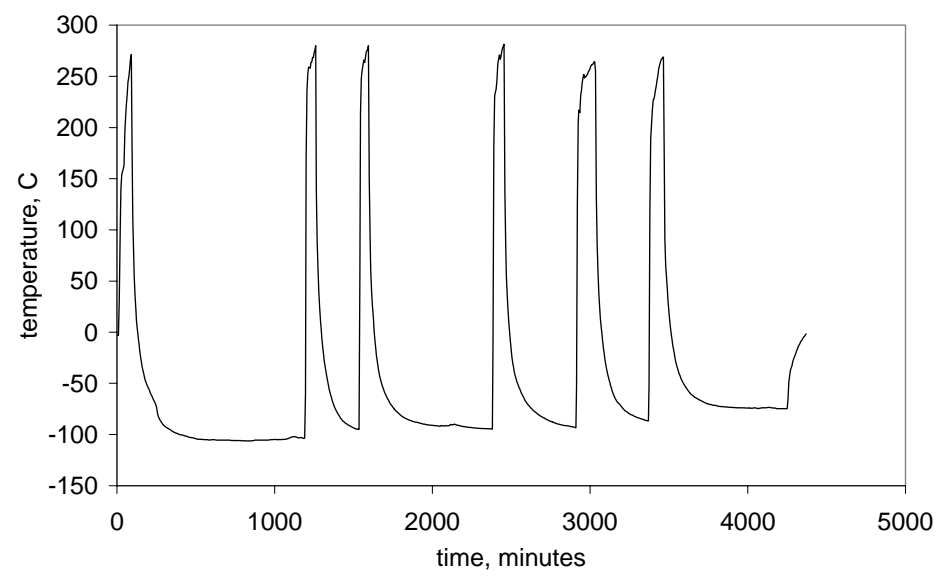

Figure 3. Example of a thermal cycling profile, from the Z-93-C55 on C-CAT composite coupon, as captured by the Keithley programmable thermometer.

The automated nature of thermal cycling was both a bane and a benefit. The programmable logic controller allowed unattended operation, allowing several thermal cycles to be accrued on each sample. Such operation was predicated on successfully meeting the high end and low end temperatures. However, owing to the unwavering power settings on the heater and low but limited cooling capacity in the cold pocket, the system was unforgiving in the case of samples that changed optically during the course of thermal cycling. In one case, the heat absorption capability of a sample, the Z-93-C55 on carbon-polyimide composite, diminished during the course of cycling, the sample did not heat to the prescribed hot end temperature and did not trip to the cold side, remaining stuck in the hot position until subsequently noticed. In several other cases, the cooling capability of the sample was diminished during the course of cycling (due to paint spalling off), and the sample remained in the cold pocket until subsequently noticed. As paint spalled off, it was found that it was necessary to soften the cold set point to allow further thermal cycling.

\section{B. Solar Absorptance and Infrared Emittance}

Initial solar absorptance for each candidate paint, along with its post thermal cycling solar absorptance, is summarized in Table 3. For the two carbon-carbon substrates coated with AZ-93 thermal cycled to a hot end temperature of $+177^{\circ} \mathrm{C}$, neither showed any substantial change in solar absorptance. The solar absorptance of the carbon-polyimide substrate coated with AZ-93 increased slightly, suggesting some change in the surface perhaps brought about by a small amount of outgassing. Outgassing of the epoxy is unlikely, because the same epoxy was used for the carbon-carbon composite samples and no change was observed in these specimens, leaving the 
polyimide suspect. One would not expect outgassing of the polyimide at $177{ }^{\circ} \mathrm{C}$, however, the change in solar absorptance from 0.123 to 0.133 is thought to be real. Inorganic white thermal control paint is particularly susceptible to minute contamination owing to the porous nature of the paint. Hence, further work is needed on the polyimide to understand its thermal properties under vacuum.

For the carbon-carbon substrates coated with Z-93-C55, a change was observed in the solar absorptance upon thermal cycling in every case. Again, outgassing of the substrate is suspect, as the hot end temperature of $277^{\circ} \mathrm{C}$ is well within the successful operating range of the paint. Indeed, the Z-93-C55 on one of the carbon-carbon composites had what appeared to be brush strokes. The source of the outgassing material is unknown but could be related to the acetone cleaning performed on the carbon-carbon composites initially. The carbon-polyimide substrate painted with the Z-93-C55 was found to spall during thermal cycling due to poor adhesion and coefficient of thermal expansion mismatch, leaving approximately half of the paint gone. The post thermal cycling solar absorptance value was great owing to the lack of paint coverage while making reflectance measurements.

For the carbon-carbon composite substrates coated with YB-71P, there was a substantial print through appearance after thermal cycling and an increase in solar absorptance similar to the Z-93-C55. The YB-71P polyimide composite combination failed completely during thermal cycling, with total de-bonding of the paint from the polyimide surface, again due to poor adhesion and coefficient of thermal expansion mismatch. No post thermal cycling solar absorptance measurements were made on the YB-71P on polyimide composite specimen.

Initial infrared emittance for each paint, along with post thermal cycling infrared emittance is summarized in Table 4. Interestingly, all three candidate paints had post thermal cycling infrared emittance values similar to their pre-thermal cycling values suggesting that the impact of suspected outgassing on infrared emittance is less than on solar absorptance. No post thermal cycling infrared emittance measurements were made on the YB-71P on polyimide composite specimen owing to complete de-bonding of the paint from the polyimide surface.

Table 3. Solar Absorptance, Before and After Thermal Cycling.

\begin{tabular}{|l|c|c|c|}
\hline & $\begin{array}{c}\text { C-CAT } \\
\text { Carbon-Carbon }\end{array}$ & $\begin{array}{c}\text { Goodrich } \\
\text { Carbon-Carbon }\end{array}$ & $\begin{array}{c}\text { GRC } \\
\text { Carbon-Polyimide }\end{array}$ \\
\hline AZ-93 & $0.123 / 0.124$ & $0.125 / 0.123$ & $0.123 / 0.133$ \\
\hline Z-93-C55 & $0.145 / 0.201$ & $0.160 / 0.368$ & $0.140 / 0.416$ \\
\hline YB-71P & $0.132 / 0.245$ & $0.082 / 0.268$ & $0.135 / \mathrm{n} / \mathrm{a}$ \\
\hline
\end{tabular}

Table 4. Infrared Emittance, at $27^{\circ} \mathrm{C}(300 \mathrm{~K})$, Before and After Thermal Cycling.

\begin{tabular}{|l|c|c|c|}
\hline & $\begin{array}{c}\text { C-CAT } \\
\text { Carbon-Carbon }\end{array}$ & $\begin{array}{c}\text { Goodrich } \\
\text { Carbon-Carbon }\end{array}$ & $\begin{array}{c}\text { GRC } \\
\text { Carbon-Polyimide }\end{array}$ \\
\hline AZ-93 & $0.955 / 0.954$ & $0.955 / 0.955$ & $0.955 / 0.955$ \\
\hline Z-93-C55 & $0.951 / 0.948$ & $0.976 / 0.971$ & $0.953 / 0.948$ \\
\hline YB-71P & $0.930 / 0.927$ & $0.937 / 0.931$ & $0.926 / \mathrm{n} / \mathrm{a}$ \\
\hline
\end{tabular}

\section{ASTM D-3359 Tape Test}

The results of the tape test are summarized in Table 5. The three specimens having the epoxy interface layer, and thermal cycled to $177^{\circ} \mathrm{C}$, were found to have only trace peeling along the parallel incisions with very little material actually removed. These represent the best thermal control paint on composite results to-date. One of the carbon-carbon specimens having an interface that was purposely roughened and painted with the Z-93-C55 had some jagged removal of material for the pristine specimen, however, the adhesion of the thermal cycled specimen

Table 5. Tape Test Results on Pristine Samples and Samples After Thermal Cycling.

\begin{tabular}{|l|c|c|c|}
\hline & $\begin{array}{c}\text { C-CAT } \\
\text { Carbon-Carbon }\end{array}$ & $\begin{array}{c}\text { Goodrich } \\
\text { Carbon-Carbon }\end{array}$ & $\begin{array}{c}\text { GRC } \\
\text { Carbon-Polyimide }\end{array}$ \\
\hline AZ-93 & 4A / 4A & 4A / 4A & 4A / 4A \\
\hline Z-93-C55 & 1A / 0A & $3 \mathrm{~A} / 0 \mathrm{~A}$ & $0 \mathrm{~A} / \mathrm{n} / \mathrm{a}$ \\
\hline YB-71P & 0A / 0A & 2A / 0A & $0 \mathrm{~A} / \mathrm{n} / \mathrm{a}$ \\
\hline
\end{tabular}


failed. The remaining specimens having the interface that was purposely roughened essentially failed the tape tests with substantial to complete removal of the paint.

\section{Future Work}

New thermal control coatings based on a binder composed of lithium silicate, not yet ready for inclusion in this study, are currently being developed via a NASA Glenn Research Center Small Business Innovative Research effort. The coefficient of thermal expansion of the lithium silicate binder has the potential to be tailored, perhaps to match the coefficient of thermal expansion of candidate composites. The lithium silicate-based thermal control paint may offer new promise for better adhesion and needs to be evaluated for thermal cycling durability in the future.

\section{Conclusions}

Carbon-carbon and carbon-polyimide composites are being considered for space radiator applications owing to their low mass and high thermal conductivity. For radiator applications where sunlight will be impinging on the surface of the radiator, it will be necessary to apply a white thermal control paint to minimize $\alpha$ and enhance $\varepsilon$. Several currently available white thermal control paints were applied to candidate carbon-carbon and carbonpolyimide coupons to identify their durability to thermal cycling. Those having an epoxy interface layer were found to be the most durable to thermal cycling, exhibiting nearly constant optical properties and very good adhesion as identified by a tape test. However, the epoxy interface layer limited the maximum thermal cycling temperature to $177^{\circ} \mathrm{C}$. Those having no interface layer were found to do poorly upon thermal cycling, with adhesion failure in a tape test.

Thermal cycling was carried out utilizing an automated system controlled by a programmable logic controller. Although the programmable logic controller controlled the operation of the thermal cycling facility successfully for coupons with unchanging optical properties, the system was found to be unable to compensate automatically for those coupons whose optical properties changed during the course of a thermal cycling exposure. For example, intervention was needed to soften set points. Additional work is needed to update the programmable logic controller to accommodate coupons with changing optical properties.

Suspected contamination riddled optical properties characterization. Although the source of contamination was not specifically pinpointed, care must be taken in the future to prepare coupons by minimizing the likelihood of contamination, including cutting and cleaning coupons in the absence of organic solvents. Additional work is needed to understand the outgassing characteristics of carbon-polyimide composites in vacuum, near the upper limit of their operating range.

New thermal control coatings being developed as part of a Small Business Innovative Research effort may be attractive for future composite applications, owing to the ability to tailor their coefficient of thermal expansion.

\section{Acknowledgments}

Acknowledgments are extended to Dr. Mrityunjay Singh, QSS Group, Inc., for his help in acquiring the carboncarbon composites and Dr. Cheryl Bowman, NASA Glenn Research Center, for kindly providing the carbonpolyimide composites.

\section{References}

${ }^{1}$ ASTM D-3359, “Standard Test Methods for Measuring Adhesion by Tape Test,” ASTM International, 100 Barr Harbor Drive, West Conshohocken, PA 19428-2959, USA, pp 1-7, 2002. 